Cite this: J. Mater. Chem. A, 2014, 2, 6504

Received 11th August 2013

Accepted 28th October 2013

DOI: 10.1039/c3ta13165c

www.rsc.org/MaterialsA

\section{Tuning the crystallinity parameters in macroporous titania films $\uparrow$}

\author{
Benjamin Mandlmeier, Norma K. Minar, Johann M. Feckl, Dina Fattakhova-Rohlfing \\ and Thomas Bein*
}

Although macroporous titania scaffolds are used for many different applications, not much is known about the importance of the synthesis strategy on the resulting materials' properties. We present a comparative study on the influence of different colloidal titania precursors for direct co-deposition with poly(methyl methacrylate) (PMMA) beads on the properties of the resulting macroporous scaffolds after calcination. The colloidal titania precursors for the film assembly differ in their size and initial crystallinity, ranging from amorphous sol-gel clusters to already crystalline pre-formed particles of $4 \mathrm{~nm}, 6 \mathrm{~nm}$ and $20 \mathrm{~nm}$ in size, as well as a combination of sol-gel and nanoparticle precursors in the so-called 'Brick and Mortar' approach. The type of the precursor greatly influences the morphology, texture and the specific crystallinity parameters of the macroporous titania scaffolds after calcination such as the size of the crystalline domains, packing density of the crystallites in the macroporous walls and interconnectivity between the crystals. Moreover, the texture and the crystallinity of the films can be tuned by postsynthesis processing of the films such as calcination at different temperatures, which can be also preceded by a hydrothermal treatment. The ability to adjust the porosity, the total surface area and the crystallinity parameters of the crystalline macroporous films by selecting suitable precursors and by applying different post-synthetic treatments provides useful tools to optimize the film properties for different applications.

\section{Introduction}

Macroporous crystalline titania offers specific morphological features such as an open porous architecture enabling efficient mass transfer, an interconnected titania framework beneficial for charge transport processes, and large pore sizes advantageous for the incorporation of bulky guest species or functional layers. ${ }^{\mathbf{1 - 4}}$ Additionally, scattering effects in the high refractive index macroporous titania scaffolds can increase the light harvesting efficiency of photovoltaic devices, and in the case of ordered arrays photonic effects can be exploited. Due to these features, macroporous titania is a very attractive system for chemical sensing of molecules, ${ }^{5,6}$ electrochemical lithium insertion, ${ }^{7,8}$ photocatalytic decomposition of organic compounds, ${ }^{9-13}$ and photoanodes in non-silicon solar cells, respectively. ${ }^{\mathbf{1 4 - 2 3}}$ In these applications, the performance is often controlled by the crystallinity and morphology of the porous titania systems. Accordingly, efforts have been made to fabricate crystalline macroporous titania morphologies by

Department of Chemistry and Center for NanoScience (CeNS), University of Munich (LMU), Butenandtstr. 11, Gerhard-Ertl-Building (E), 81377 Munich, Germany. E-mail: bein@lmu.de; Fax: +49 (0)89 2180 77622; Tel: +49 (0)89 218077621 $\dagger$ Electronic supplementary information (ESI) available: SEM, TEM and optical microscopy images of macroporous titania samples and information on particle sizes. See DOI: 10.1039/c3ta13165c bottom-up self-assembly approaches. Ordered macroporous titania is usually made using spherical polymer beads as structural templates. Typical protocols involve either infiltration of the voids of ordered bead arrays with different titania precursors (the so-called infiltration method involving two main steps, the bead assembly and their subsequent impregnation with suitable titania precursors), ${ }^{\mathbf{2 0 2 2 - 2 8}}$ or a direct coassembly of the titania precursors and the polymer beads (the so-called co-deposition method). ${ }^{29-34}$ The polymer template is removed after the solidification of the titania phase, leaving a porous titania replica. Either amorphous sol-gel derived titania or already crystalline pre-formed titania nanoparticles are used as titania precursors..$^{35-51}$ The crystallinity of the resulting macroporous titania morphologies can differ widely for the various synthesis approaches. Key properties such as phase composition, crystal shape, crystal size and interconnectivity of the crystals strongly depend on synthesis parameters such as the choice of the titania precursors or the subsequent thermal treatment. Additional features of the macroporous films such as electron mobility, pore connectivity via small interconnection windows and/or porous walls, surface area, as well as pore size, thickness and homogeneity of the films or their optical behavior are expected to have a great impact on the performance of such films in different devices. 
In order to address these issues we have used several titania precursors to compare their influence on the properties of the resulting macroporous films. The basic approach involves onepot fabrication of crystalline macroporous titania films using direct co-deposition of colloidal titania precursors and poly(methyl methacrylate) (PMMA) beads. Although the co-deposition methods can offer several advantages such as better control over the precursor-to-template ratios, simplicity and shorter fabrication times, they have not been studied as widely as the infiltration methods.

We have examined various colloidal titania precursors for the film assembly, differing in their size and initial crystallinity and ranging from amorphous sol-gel clusters ${ }^{52}$ to already crystalline pre-formed particles with a size of $4 \mathrm{~nm},{ }^{44} 6 \mathrm{~nm}^{53}$ and $18 \mathrm{~nm} .^{54}$ These particles can be viewed as representatives of precursors used for different titania nanostructures. Additionally, a combination of sol-gel and nanoparticle precursors according to the so-called 'Brick and Mortar' approach was studied. ${ }^{42}$ Moreover, we have investigated the influence of post-synthetic treatments of the assembled films on the crystallization behavior and final properties. As such, thermal treatments at different temperatures $\left(400-500{ }^{\circ} \mathrm{C}\right)$ and a hydrothermal treatment of the PMMA-titania composites before calcination were explored. This last treatment has a profound influence on the thermally induced growth of the nanocrystalline titania and the shrinkage of the structures, thus stabilizing the desired porous scaffolds.

\section{Experimental}

\section{Chemicals}

The commercially available chemicals were used without further purification. Methyl methacrylate (99\%, MMA), sodium dodecyl sulfate (SDS), tetraethyl orthotitanate, titanium tetraisopropoxide, titanium tetrachloride, hydrochloric acid (conc.), acetic acid (conc.), nitric acid (conc.), potassium chloride and the organic solvents were obtained from Sigma-Aldrich. Pure water was obtained from a Millipore $\mathrm{Q}$ system.

\section{Synthesis of colloidal poly(methyl methacrylate) nanoparticles}

PMMA spheres were synthesized via an emulsion polymerization route, ${ }^{55}$ yielding an aqueous colloidal solution. Clean water (98.0 mL, Millipore Q) was degassed with nitrogen in a threenecked flask $(250 \mathrm{~mL})$ for 1 hour, before sodium dodecyl sulfate (5.6 mg, $0.017 \mathrm{mmol}$, SDS) and methyl methacrylate (35.5 g, 0.35 mmol, MMA) were added. The monomer mixture was stirred for 1 hour at $90{ }^{\circ} \mathrm{C}$ in a nitrogen atmosphere to obtain a homogeneous emulsion. A solution of potassium peroxodisulfate (56 mg, $0.2 \mathrm{mmol}, \mathrm{K}_{2} \mathrm{~S}_{2} \mathrm{O}_{8}$ ) in water $(2 \mathrm{~mL}$ ) was injected to initiate the polymerization. The solution turned white within 5 minutes, indicating the formation of polymer particles. The reaction was continued for 2 hours at $90{ }^{\circ} \mathrm{C}$, before the flask was exposed to air and cooled with an ice bath. The colloidal solution was stirred overnight, filtered through glass wool and washed three times by centrifugation (50 000 rcf; 30 minutes) and redispersion in water.
The PMMA was dried and redispersed in ethanol to prepare mixtures with various titania precursors. The size of the PMMA spheres was estimated to be around $320 \mathrm{~nm}$ from FE-SEM images, which is in good agreement with the average size of $324 \mathrm{~nm}$ measured by dynamic light-scattering (DLS) (Fig. S1a and b†).

\section{Synthesis of macroporous titania}

All the following mixtures contained $325 \mathrm{mg}$ of ground PMMA powder, and the amount of titania precursors was adjusted to transform into $100 \mathrm{mg}$ anatase after calcination.

\section{Sol-gel}

The titania sol-gel solution (SG) corresponds to the one we have used before for dip-coating infiltration of PMMA colloidal crystal template films. ${ }^{52} 325 \mathrm{mg}$ PMMA powder was well dispersed in $4 \mathrm{~mL}$ ethanol by using an ultrasonic bath for several hours. Tetraethyl orthotitanate ( $0.36 \mathrm{~g}, 1.25 \mathrm{mmol}$, TEOT) was added and the solution was stirred for another hour at room temperature. To this mixture $230 \mu \mathrm{L}(37 \%, 2.8 \mathrm{mmol})$ of $\mathrm{HCl}$ was added. The solution was ready for use after additional stirring for 10 minutes. We note that direct addition of the prehydrolyzed TEOT solution to the PMMA solution led to a strong increase of viscosity, which would be unfavorable for further use.

\section{NP-20}

Crystalline anatase nanoparticles were prepared according to literature reports. ${ }^{56} 1.90 \mathrm{~mL}$ acetic acid $(33.0 \mathrm{mmol})$ was added dropwise to $9.47 \mathrm{~g}$ titanium tetraisopropoxide $(33.0 \mathrm{mmol})$ within 15 minutes under stirring at room temperature. To the faint-yellow solution $48.5 \mathrm{~mL}$ water was added to form a white precipitate. The mixture was stirred for 1 hour before $4 \mathrm{~mL}$ of nitric acid (conc.) was added. Afterwards this mixture was refluxed at $80{ }^{\circ} \mathrm{C}$ for 75 minutes. Prior to the hydrothermal treatment in an autoclave (100 mL, PTFE) at $250{ }^{\circ} \mathrm{C}$ for 12 hours, $0.44 \mathrm{~mL}$ of water was added. After reaction, $0.40 \mathrm{~mL}$ of $65 \%$ nitric acid was added to the precipitate, which was treated with ultrasound for 60 minutes. The resulting solution was concentrated by rotary evaporation and the titania nanoparticles were washed three times with absolute EtOH by centrifugation and redispersion (50 $000 \mathrm{rcf} ; 20$ minutes). The particles washed in this way were dried at room temperature.

For film preparation (NP-20), $100 \mathrm{mg}$ of the dried titania NPs were redispersed in $4 \mathrm{~mL}$ absolute ethanol by ultrasonic treatment, then $325 \mathrm{mg}$ of fine PMMA particle powder was added, and the solution was stirred overnight.

\section{NP-6}

Titania nanoparticles (NP-6) were synthesized by a route described by Niederberger et al. ${ }^{42,53} 1.5 \mathrm{~mL}$ of titanium tetrachloride $(13.7 \mathrm{mmol})$ was cautiously dropped into $10 \mathrm{~mL}$ of toluene under stirring. Then this solution was added to $30 \mathrm{~mL}$ benzyl alcohol (290.8 $\mathrm{mmol})$, which was further heated in a plastic autoclave at $60{ }^{\circ} \mathrm{C}$ for 20 hours. After the reaction, the nanoparticle solution was centrifuged (50 000 rcf; 30 minutes) and the titania content within the pellet was determined by 
thermogravimetric analysis. Finally, $100 \mathrm{mg}$ of titania nanoparticles were redispersed in $4 \mathrm{~mL}$ ethanol and used for film formation as described for NP-20.

\section{NP-4}

The preparation is similar to that of NP-6, but in contrast, $30 \mathrm{~mL}$ of tert-butyl alcohol (320 mmol) was used instead of benzyl alcohol. ${ }^{44}$ A twofold microwave heating procedure (Synthos 3000, Anton Paar) was performed in $100 \mathrm{~mL}$ autoclaves. One step involved heating up to $80^{\circ} \mathrm{C}$ within one minute, followed by cooling to and dwelling at $50{ }^{\circ} \mathrm{C}$ for another 20 minutes. The resulting titania nanoparticle solution was washed by a successive particle flocculation, using a mixture of $n$-heptane/ tert-butyl alcohol (2:1, vol.), and centrifugation (50 000 rcf; 15 minutes). The final particles were redispersed in absolute ethanol, and the titania content was estimated by thermogravimetric analysis. Typically, $100 \mathrm{mg}$ of titania nanoparticles (NP4) were dispersed in $1 \mathrm{~mL}$ ethanol and added to $3 \mathrm{~mL}$ of an ethanolic PMMA particle (325 mg) dispersion. Prior to use, the final solution was stirred overnight.

\section{NP-20 with sol-gel ('Brick and Mortar')}

This titania precursor mixture was prepared by mixing $70 \mathrm{mg}$ nanoparticles (NP-20) and $325 \mathrm{mg}$ PMMA particles in $4 \mathrm{~mL}$ of ethanol. The mixture was stirred overnight, afterwards $108 \mathrm{mg}$ of TEOT $(0.37 \mathrm{mmol})$ was added. The solution was stirred for 1 hour at room temperature and finally $69 \mu \mathrm{L}$ of $\mathrm{HCl}(37 \%$, $0.8 \mathrm{mmol}$ ) was added.

\section{Film preparation}

Typically, all PMMA/titania precursor solutions were spincoated $(2000 \mathrm{rpm})$ or drop-cast on a silicon wafer $(15 \mu \mathrm{L}$ on $2.25 \mathrm{~cm}^{2}$ ). After drying at room temperature under vibrationfree conditions (ca. 1 day), films were calcined in air at 400, 450 and $500{ }^{\circ} \mathrm{C}$, respectively. The heating ramp was $1^{\circ} \mathrm{C} \mathrm{min}-1$ and the dwell time was 1 hour.

\section{Humidity treatment}

PMMA/NP-6 composite films were also treated according to a procedure reported elsewhere. ${ }^{51}$ First they were dried in air at room temperature, then stored in a desiccator and exposed to water vapor at $100{ }^{\circ} \mathrm{C}$ for 7 days, by placing the desiccator in an oven at this temperature. The bottom of the desiccator was filled with a saturated aqueous $\mathrm{KCl}$ solution. Afterwards calcination at $450{ }^{\circ} \mathrm{C}$ in air was applied. The heating ramp was $1{ }^{\circ} \mathrm{C}$ $\min ^{-1}$ and the dwell time was 1 hour.

\section{Results}

We have used several titania precursors with different crystallinity parameters and crystal sizes in combination with $320 \mathrm{~nm}$ spherical PMMA templates (Fig. S1, ESI $\dagger$ ) to fabricate macroporous films in a co-deposition process by spin-coating. One of the most common precursors for the assembly of titania morphologies is amorphous colloidal sol-gel titania (denoted as SG). ${ }^{\mathbf{1 3} \mathbf{1 4 , 3 5 , 3 9}}$ We have used this precursor system as a known reference for the fabrication of films. The SG precursor was prepared by adding tetraethyl orthotitanate (TEOT) and $\mathrm{HCl}$ to a dispersion of PMMA beads in ethanol. Additionally, we have examined colloidal dispersions of pre-formed crystalline titania nanoparticles with three different particle sizes. The smallest particles (denoted as NP-4) were prepared by a microwaveassisted synthesis in tert-butanol. ${ }^{\mathbf{4 4}}$ The particles prepared in this way have a size of about 3-4 nm with a narrow size distribution, they are largely crystalline corresponding to the anatase phase, and are well dispersible in ethanol (Fig. S2a and $\mathrm{b}^{\dagger} \dagger$ ). Slightly larger particles of $c a$. 5-6 nm in size (assigned NP-6) were obtained by a solvothermal synthesis in benzyl alcohol. ${ }^{\mathbf{4 2 , 5 3}}$ These particles are poorly dispersible in ethanol resulting in turbid dispersions of agglomerated nanoparticles (Fig. S2c and $\mathrm{d} \dagger)$. The largest fully crystalline anatase particles of $c a .20 \mathrm{~nm}$ in size (denoted as NP-20) were prepared by a hydrothermal treatment of hydrolyzed tetraisopropyl orthotitanate (Fig. S3a and b). ${ }^{56,57}$

All the macroporous titania films described in this work were prepared by a similar procedure. Typically, ethanolic dispersions of titania precursors were mixed with an ethanolic dispersion of the PMMA beads at $\mathrm{TiO}_{2} / \mathrm{PMMA}$ weight ratio of 1 to 3.25. The resulting white mixtures were spin-coated or dropcast on glass substrates and the composite films were heated in air at $400-500{ }^{\circ} \mathrm{C}$. A similar heat treatment allows for a direct unambiguous evaluation of the role of different precursors in the crystallization process and the final crystallinity of the films.

All the films are semitransparent, microscopically rough and slightly scattering after calcination, and exhibit an average thickness of about $1 \mu \mathrm{m}$. The thickest films are obtained with the amorphous SG precursor, while the use of a larger crystalline NP-20 precursor results in the thinnest films. Although the films obtained from different precursors look similar at the first glance, they show a striking difference in their mechanical stability and adhesion to the substrate. The SG precursor provides homogeneous coatings with good adhesion to the substrate. The coatings become more fragile with increasing crystal size of the crystalline precursors. The films assembled from NP-4 and NP-6 still have rather good mechanical stability and substrate coverage, but the films obtained from NP-20 are very fragile and do not cover the substrate completely. Thicker NP-20 films, prepared by drop-casting, strongly contract after drying into single small flakes lying loosely on the substrate (Fig. S4†).

SEM images (Fig. 1) demonstrate that the films assembled from all the precursors examined here have an open macroporous morphology with a uniform pore size but rather low periodicity of the pore ordering. The specific parameters of porosity such as pore size, pore connectivity, wall thickness and, importantly, the change in the pore size (shrinkage) after calcination strongly depend on the origin of the used precursors. The sol-gel sample SG (Fig. 1a) has the highest degree of pore ordering. This scaffold shows individual macropores with diameters of about $235 \mathrm{~nm}$ that are interconnected by windows sized 50-120 nm. The titania walls are dense and compact, with a thickness of about 20-40 $\mathrm{nm}$. The morphology obtained by 

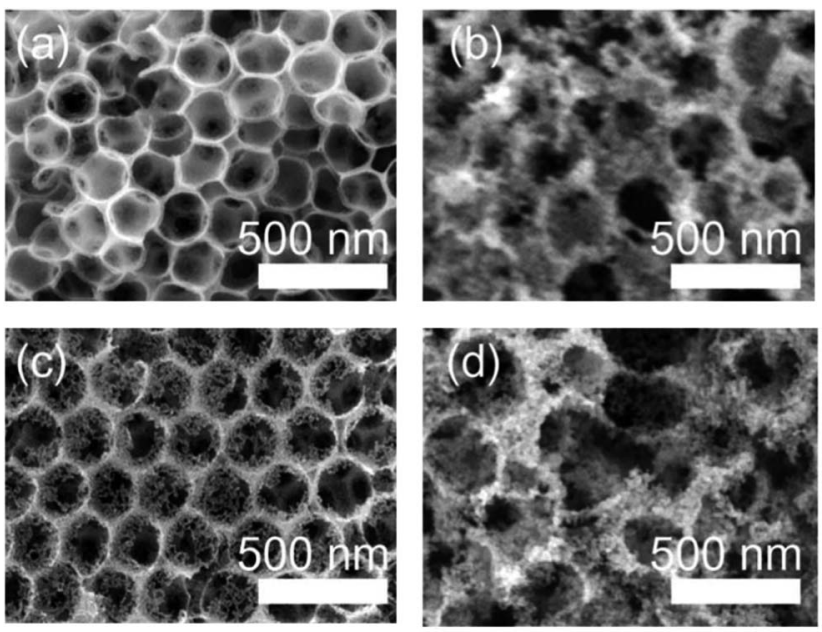

Fig. 1 Top view SEM images of calcined $\left(500^{\circ} \mathrm{C}\right)$ macroporous $\mathrm{TiO}_{2}$ films assembled from $320 \mathrm{~nm}$ PMMA spheres and different titania precursors: (a) TEOT based hydrolyzed sol-gel solution (SG) and anatase nanoparticles: (b) NP-4, (c) NP-6, and (d) NP-20.

this one-pot co-precipitation is in fact very similar to that obtained by a two-step infiltration approach, but its fabrication is simpler and less time-consuming. ${ }^{52}$

When crystalline nanoparticulate building blocks are used instead of the amorphous molecular precursor, the size of the individual crystals affects the properties of the resulting porous systems. In contrast to the smooth and dense walls of the SG sample, the walls of all nanoparticle samples (NP-4, NP-6, and NP-20) show a pronounced texture resulting from packing of the crystalline solid building blocks. Basically, the bigger the size of the used particles, the less perfect is the replication of the curvature of the original PMMA beads. The films assembled from small NP-4 and NP-6 precursor crystals (Fig. 1b and c) show less periodic pore ordering compared to the SG sample, but much better regularity compared to films obtained from the large crystalline NP-20 precursor (Fig. 1d). Surprisingly, the films assembled from the agglomerated NP-6 particles show more regular pore packing compared to the smaller dispersible NP-4 precursor derived films, which are rather rough and irregular. Samples SG and NP-4 have a similar pore size of around $240 \mathrm{~nm}$, which corresponds to a shrinkage with respect to the original template size of $320 \mathrm{~nm}$ by about $25 \%$. The NP-6 films show a rather regular pore packing comparable to that of the sol-gel sample, but also a larger pore diameter of 270 to $290 \mathrm{~nm}$ and a significantly reduced shrinkage of about $10-16 \%$. In contrast, the NP-20 sample assembled from large crystalline nanoparticles is quite rough, disordered and is composed of non-uniform pores and walls with a broad variety in shape and size (Fig. 1d). The shrinkage of the pore size is further reduced to approximately 3 to $10 \%$.

The wetting and adhesion properties of the crystalline precursor dispersions are drastically improved when some amount of sol-gel titania is added to the nanocrystal dispersion. This so-called 'Brick and Mortar' approach developed by us previously for block-copolymer-templated mesoporous titania assembled from nanoparticles was shown to be a very promising method to obtain fully crystalline porous layers at relatively low temperatures. ${ }^{42}$

This approach also seems to be applicable to larger pore dimensions and to other types of templates. We view this strategy as a successful extension to a large-scale hard templating method and to much larger $\mathrm{TiO}_{2}$ precursor nanoparticles. Thus, mixing of the NP-20 particle dispersion (crystalline brick) with some amount of SG precursor (mortar) at the titanium ratio of $70: 30 \mathrm{wt} \%$ (NP-20:SG) drastically changes the properties of the resulting films (denoted as NP-20-SG). The films completely cover the substrate, and they have a much better adhesion and mechanical stability than the pure NP-20 films (Fig. S4 $\dagger$ ). The highly accessible morphology of the NP-20SG film resembles that of the SG films with well-formed uniform pores, a smooth surface and dense interconnecting walls (Fig. 2a and b). In contrast, in the NP-20 sample (Fig. 2b) the pores are only partially ordered with a size ranging from 250 to $270 \mathrm{~nm}$ and the structure features rather inhomogeneous wall thicknesses, which is attributed to loosely packed particles after sintering at $500{ }^{\circ} \mathrm{C}$.

Besides the morphology, the crystallinity of the final scaffold is often crucial; the latter can be easily and significantly influenced by the choice and the treatment of the precursor. In order to investigate the effect of different heating protocols on the development of the titania precursors, we performed a series of SEM, HR-TEM, SAED (Fig. 3, S3 and S5 $\dagger$ ) and XRD measurements (Fig. 4 and Table 1). Regardless of the initial crystallinity of the used titania precursor, all films are crystalline after calcination beyond $400{ }^{\circ} \mathrm{C}$ ( 1 hour in air). However, the specific parameters of crystallinity (grain size and connectivity) and the thermally induced changes in crystallinity (grain growth, texture) differ strongly with the type of the precursor.

A significant change of the crystal size with temperature $\left(400{ }^{\circ} \mathrm{C}\right.$ and $500{ }^{\circ} \mathrm{C}$ ) was observed for macroporous films containing small NP-4 (Fig. $3 \mathrm{a}$ and $\mathrm{b}$ and $4 \mathrm{a}$ ) and NP-6 (Fig. $3 \mathrm{c}$ and $\mathrm{d}$ and $4 \mathrm{c}$ ) nanoparticles, respectively (see also Table S1†). The SAED patterns indicate the increase in crystal size with higher calcination temperatures induced by solid state diffusion and sintering of the small nanoparticulate precursors. Both samples calcined at $500{ }^{\circ} \mathrm{C}$ show the presence of bright spots in addition to the rings due to the transformation of small randomly oriented particles into larger crystalline anatase domains, which is consistent with reported data already shown for NP-6 nanoparticles. ${ }^{\mathbf{4 2 5 3}}$ In comparison, NP-20 exhibits nearly no
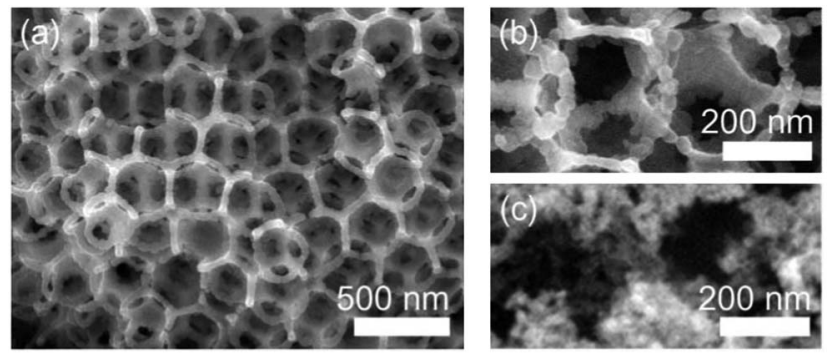

Fig. 2 Top view SEM images of SG-NP-20-SG ( $a$ and b) and NP-20 (c). 

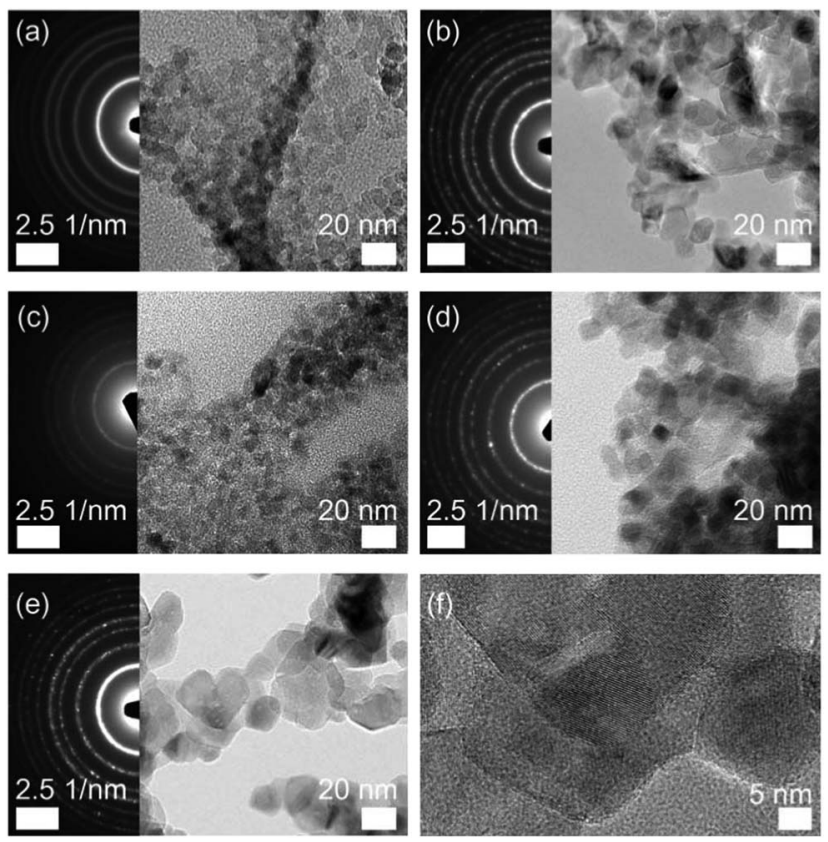

Fig. 3 Selected area electron diffraction (SAED) patterns of larger sample areas and TEM images of films removed from the substrate after calcination: (a) NP-4 $\left(400^{\circ} \mathrm{C}\right),\left(\right.$ b) NP-4 $\left(500^{\circ} \mathrm{C}\right),(\mathrm{c}) \mathrm{NP}-6(400$ ${ }^{\circ} \mathrm{C}$ ), (d) NP-6 $\left(500^{\circ} \mathrm{C}\right)$ and (e and f) NP-20-SG $\left(500^{\circ} \mathrm{C}\right)$ at different magnifications.
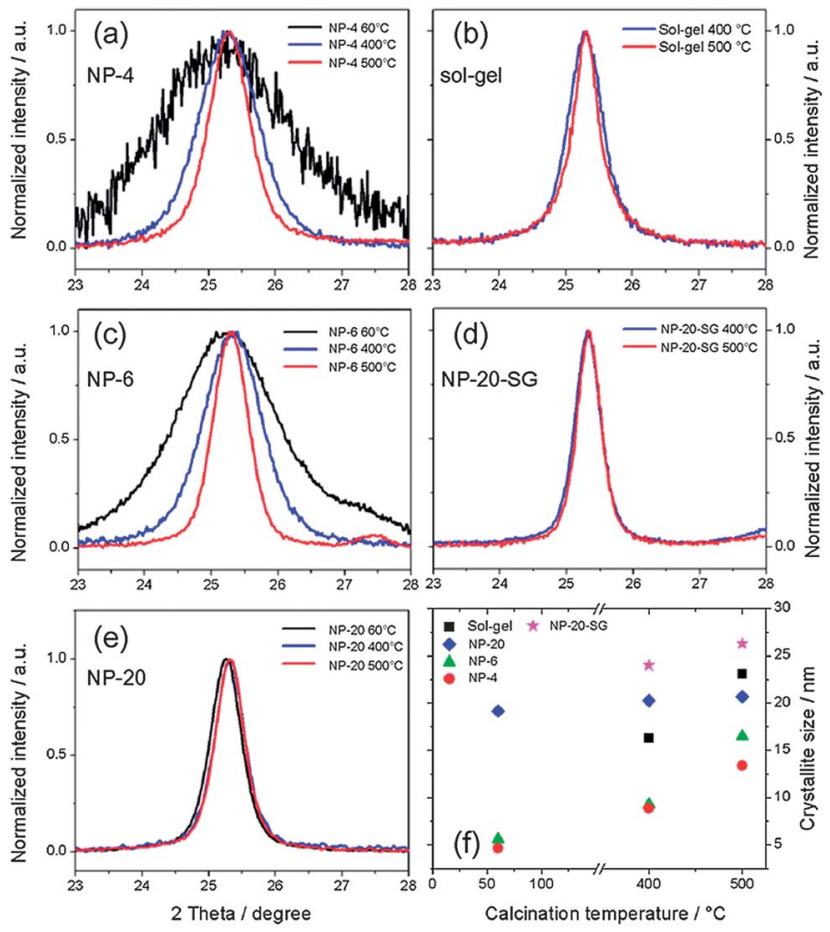

Fig. 4 XRD study showing anatase (101) reflections of macroporous titania samples after different treatments (after drying at $60{ }^{\circ} \mathrm{C}$ for 1 hour and calcination). The normalized reflections of macroporous titania samples obtained with nanocrystalline building blocks NP-4, NP-6 and NP-20 are shown in (a), (c) and (e), respectively. SG and NP20-SG are shown in (b) and (d), respectively. (f) Overview of the crystallite domain size of all samples (determined with the Scherrer equation from the line broadening of the 101 anatase reflection). heating induced grain growth, which is attributed to the high crystallinity and the larger initial size of the crystals (Fig. $4 \mathrm{f}$ and S3 $†$ ). ${ }^{56,57}$ However, the crystal size increases after calcination for the NP-20-SG sample ('Brick and Mortar') containing some amount of titania sol-gel precursor in addition to the NP-20 particles. The sol-gel precursor itself shows consistent heatinginduced grain growth (Fig. 4b), which has two effects in the sample NP-20-SG. First, after heating at $400{ }^{\circ} \mathrm{C}$ the domains are about $18 \%$ larger than for pure NP-20 and after heating at $500{ }^{\circ} \mathrm{C}$ an additional growth up to $26 \mathrm{~nm}$ is observed, while the crystal size in pure NP-20 is almost not affected by the heat treatment (Fig. 4f). The walls of the calcined sample NP-20-SG are highly crystalline without any indication of an amorphous phase (Fig. 3e). Therefore, we can conclude that the already crystalline NP-20 particles (70 wt $\% \mathrm{Ti})$ assist in the crystallization of the initially amorphous molecular precursor $(30 \mathrm{wt} \% \mathrm{Ti}$ ) located around the crystalline seeds. This is similar to the effects observed in our previous studies of mesoporous titania. ${ }^{42,43,52}$ Moreover, the presence of the amorphous precursor leads to a better connectivity of the titania crystals in the walls and to a reduced interparticulate textural porosity (Fig. 3e and f).

The properties of the different macroporous samples are summarized in Table 1.

\section{Enhancing crystallization through processing}

In addition to the choice of the precursor and the thermal treatment as a means to tune the crystallinity and the morphology of the films, we have investigated an alternative strategy to control the final crystal size, morphology and macroscopic film quality. For that purpose, an additional postsynthetic hydrothermal treatment (also known as Delayed Humidity Treatment, DHT) of the assembled PMMA/titania composites was performed prior to the calcination of the films.

Identical films assembled of NP-6 nanoparticles were treated differently before calcination in air at $450{ }^{\circ} \mathrm{C}$ (Fig. $5 \mathrm{a}-\mathrm{c}$ ). One sample was calcined directly after film assembly and drying at $60{ }^{\circ} \mathrm{C}$ (1 hour) (NP-6), while the other sample was kept for 7 days at $100{ }^{\circ} \mathrm{C}$ at around $90 \%$ relative humidity (NP-6-DHT). As shown in the TEM images, the hydrothermal treatment at elevated temperatures inhibits crystal growth and leads to a slightly smaller particle size of $10 \pm 2 \mathrm{~nm}$ after calcination at $450{ }^{\circ} \mathrm{C}$ (Fig. 5b) compared to the non-treated films $(12 \pm 3 \mathrm{~nm}$, Fig. 5c). Strikingly, the macrostructural order was significantly improved upon applying the DHT, and accompanied by a strong reduction of the temperature-induced shrinkage. The crystalline titania morphology obtained after calcination of the NP-6DHT film at $450{ }^{\circ} \mathrm{C}$ is a well-ordered replication of the inverse structure of the PMMA spheres, which indicates good adhesion of the nanoparticles to the template surface. The architecture, appearing almost like an array of titania hollow spheres rather than a macroporous scaffold, is also strongly stabilized against heating-induced shrinkage. The corresponding TEM image in Fig. 5a shows wall to wall distances of around $315 \mathrm{~nm}$, which is in the same range as the size of the initial PMMA particles ( $2320 \mathrm{~nm}$, Fig 5a (inset), Fig S1†). 
Table 1 Characteristics of macroporous titania films treated at different temperatures

Grain size ${ }^{a}(\mathrm{~nm})$ and percentage increase compared to the initial precursor size

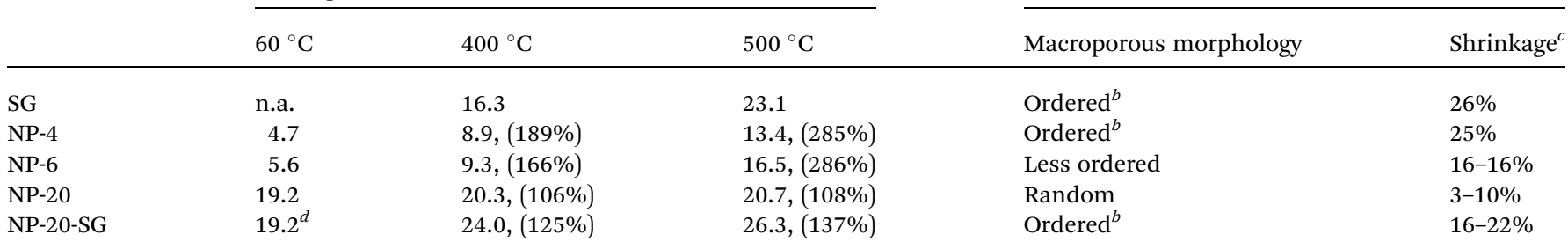

${ }^{a}$ Estimated from the broadening of the 101 anatase reflection in XRD patterns using the Scherrer equation. ${ }^{b}$ Locally ordered domains; no photonic effects were observed. ${ }^{c}$ Difference of the macropore diameter in relation to the initial PMMA sphere size of $c a .320 \mathrm{~nm}$ after calcination. ${ }^{d}$ Assuming an equal size as shown in sample NP-20.
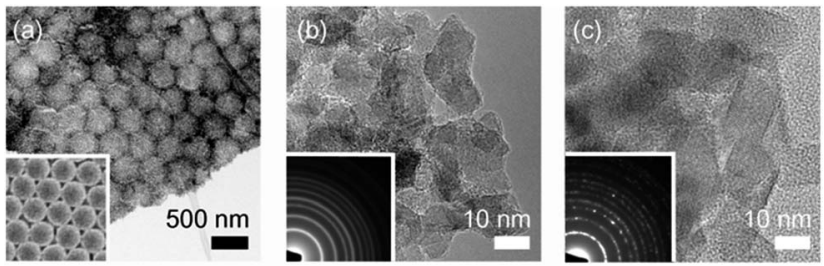

Fig. 5 ( $a$ and b) TEM images at different magnifications of a NP-6-DHT derived network after post-synthetic humidity treatment (DHT) followed by calcination at $450^{\circ} \mathrm{C}$. The inset in (a) shows an SEM image of the PMMA templates at the same magnification. HR-TEM images and SAED patterns (insets) show differences in crystallinity between NP-6$\mathrm{DHT}$ (b) and the untreated NP-6 reference (c).

\section{Discussion}

As we have demonstrated above, crystalline macroporous titania films can be easily prepared by a one-step co-deposition procedure from a colloidal mixture of PMMA beads and titania precursors. Various titania building blocks, although being very different in size and the degree of crystallinity, can be assembled with the spherical PMMA beads. The overall porous morphology of the resulting films is determined by the PMMA beads, being a negative replica of the close-packed bead arrays. However, the microscopic features of the films are governed by the choice of the titania building blocks. We tentatively attribute this effect to different hydrophilicity/hydrophobicity of the particles obtained by different reactions, and consequently different interactions with the PMMA beads. The amorphous sol-gel derived titania species are most capable of replicating the PMMA bead arrays, resulting in the best periodicity of the macroscopic pore system. Moreover, the films assembled from the amorphous precursors are smooth, and they feature good mechanical stability and good adhesion to the substrate. However, the sol-gel derived films are prone to strong volume changes and shrinkage due to densification upon crystallization of the titania scaffold during the high-temperature calcination. Because of that, only relatively thin films can be prepared without cracking and delamination of the titania coating.

This undesired shrinkage is greatly reduced when larger and highly crystalline titania nanoparticles are used for the film assembly. However, the films prepared in this way have a lower order of the pore packing, a lower mechanical stability and inferior adhesion to the substrate, which becomes more pronounced for larger crystalline building blocks. The advantages of both strategies can be combined by blending amorphous and crystalline titania species for the film assembly in a 'Brick and Mortar' approach. An addition of about $30 \mathrm{wt} \%$ of amorphous precursor to the crystalline particles is sufficient to obtain rather regular macroporous films with good adhesion to the substrate, largely reduced shrinkage and increased thickness.

An important issue in the fabrication of porous titania coatings is the crystallinity of the resulting titania scaffold. As we have demonstrated above, all the films are fully crystalline at temperatures beyond $400{ }^{\circ} \mathrm{C}$, independent of the initial crystallinity of the titania precursor. However, the specific crystallinity parameters such as the size of the crystalline domains, packing density of the crystallites in the macroporous walls and interconnectivity between the crystals strongly depend on the type of the precursor. The larger the size and the higher the crystallinity of the building blocks used for the film assembly, the less dense is the packing of the titania crystals in the macroporous titania scaffold after calcination. The walls of the films obtained from the amorphous precursor are smooth and dense. In contrast, the walls of all nanoparticulate samples bear a textural porosity due to the crystalline solid building blocks.

In addition to the choice of the precursor, the texture and the crystallinity of the films can be tuned by post-synthesis processing of the films such as calcination at different temperatures, which can be also preceded by a hydrothermal treatment. Generally, we conclude that the temperature-induced relative change in size of the crystalline domains in the titania films inversely correlates with the size of the precursors used for the film assembly. The smallest change is observed for the largest crystalline NP-20 precursor, which practically does not grow during calcination. The largest relative change in the crystal size after thermal treatment is observed for the initially amorphous SG precursor, which gives $16 \mathrm{~nm}$ and $23 \mathrm{~nm}$ large crystals after calcination at $400{ }^{\circ} \mathrm{C}$ and $500{ }^{\circ} \mathrm{C}$, respectively, and for small NP4 nanoparticles whose size changes from initially $3-4 \mathrm{~nm}$ to $9 \mathrm{~nm}$ and $13 \mathrm{~nm}$ at $400{ }^{\circ} \mathrm{C}$ and $500{ }^{\circ} \mathrm{C}$, respectively. A similar trend was observed for NP-6 (Table 1). 
This temperature-induced change in crystallinity has a great impact on the transport properties of the titania scaffold. We have already performed a time-resolved terahertz spectroscopy study of the electron mobility of several of the titania films described in this communication, namely the SG films assembled from the amorphous precursors, the NP-20 films assembled from the large crystalline nanoparticles and the 'Brick and Mortar' SG-NP-20 films, all of them being calcined at $400{ }^{\circ} \mathrm{C} .5^{58}$ The results demonstrate that the conductivity of the walls composed of initially crystalline NP-20 nanoparticles is rather poor after calcination, being dominated by a hopping mechanism. In contrast, the crystalline phase obtained after calcination of the initially amorphous SG precursor at $400{ }^{\circ} \mathrm{C}$ has the highest conductivity with some contribution of a band-like transport. Finally, the properties of the SG-NP-20 films lie somewhere between the properties of the SG and NP-20 samples. These observations illustrate that the mobility and the underlying conduction mechanism of the microscopic mobility of the crystalline titania scaffolds are strongly dependent on the film fabrication method. Therefore, the ability to adjust the porosity, the total surface area and the crystallinity parameters of the crystalline macroporous films by selecting suitable precursors and by applying different post-synthetic treatments can provide a useful tool to optimize the film properties according to the needs of the applications.

\section{Conclusions}

In this study we have examined the crystallization behavior of titanium dioxide in macroporous thin films as a function of different precursors and temperature treatments. These macroporous structures were prepared by co-deposition of PMMA spheres with a variety of titania precursors ranging from amorphous sol-gel, differently sized crystalline titania nanoparticles and a combination of both. The precursors show characteristic crystal growth upon calcination at 400, 450 and $500{ }^{\circ} \mathrm{C}$ with crystalline domains ranging from around $4 \mathrm{~nm}$ up to $26 \mathrm{~nm}$. A post-synthetic humidity treatment drastically affects the crystal growth and reduces the shrinkage that otherwise occurs in polymer hard-templated systems. This method allows us to inhibit crystal growth that would otherwise occur at elevated calcination temperatures above $400{ }^{\circ} \mathrm{C}$.

In conclusion, we present a comparative study on the crystallization of macroporous titania that examines phase composition, crystal shape, crystal size and interconnectivity of the crystals in macroporous frameworks. Additionally, mesoscopic features of the macroporous films such as porosity, pore size, pore connectivity and interparticle texture, as well as macroscopic properties such as thickness and homogeneity of the films are addressed that are expected to have a great impact on the performance of such films in different devices.

\section{Acknowledgements}

Financial support from the Bavarian research network SolTech and from the DFG SPP program 1613 is gratefully acknowledged. The authors thank Dr Steffen Schmidt for TEM measurements.

\section{Notes and references}

1 B. T. Holland, C. F. Blanford and A. Stein, Science, 1998, 281, 538.

2 B. T. Holland, C. F. Blanford, T. Do and A. Stein, Chem. Mater., 1999, 11, 795.

3 M. E. Turner, T. J. Trentler and V. L. Colvin, Adv. Mater., 2001, 13, 180.

4 J. E. G. J. Wijnhoven, L. Bechger and W. L. Vos, Chem. Mater., 2001, 13, 4486.

5 D. Chandra, M. Bekki, M. Nakamura, S. Sonezaki, T. Ohji, K. Kato and T. Kimura, J. Mater. Chem., 2011, 21, 5738.

6 J. Li, X. Zhao, H. Wei, Z.-Z. Gu and Z. Lu, Anal. Chim. Acta, 2008, 625, 63.

7 L. Kavan, M. T. Zukalova, M. Kalbac and M. Graetzel, J. Electrochem. Soc., 2004, 151, A1301.

8 H. Kim, M. G. Kim and J. Cho, Adv. Energy Mater., 2012, 2, 1425.

9 A. Natoli, A. Cabeza, Á. G. De La Torre, M. A. G. Aranda and I. Santacruz, J. Am. Ceram. Soc., 2012, 95, 502.

10 M. Wu, Y. Li, Z. Deng and B.-L. Su, ChemSusChem, 2011, 4, 1481.

11 H. Chen, C. E. Nanayakkara and V. H. Grassian, Chem. Rev., 2012, 112, 5919.

12 M. Lim, Y. Zhou, B. Wood, Y. Guo, L. Wang, V. Rudolph and G. Lu, J. Phys. Chem. C, 2008, 112, 19655.

13 J. I. L. Chen, G. von Freymann, S. Y. Choi, V. Kitaev and G. A. Ozin, Adv. Mater., 2006, 18, 1915.

14 L. I. Halaoui, N. M. Abrams and T. E. Mallouk, J. Phys. Chem. $B, 2005,109,6334$.

15 A. Mihi, M. E. Calvo, J. A. Anta and H. Miguez, J. Phys. Chem. C, 2008, 112, 13.

16 S. Colodrero, A. Mihi, J. A. Anta, M. Ocana and H. Miguez, J. Phys. Chem. C, 2009, 113, 1150.

17 A. Mihi, C. Zhang and P. V. Braun, Angew. Chem., Int. Ed., 2011, 50, 5711.

18 S. Nishimura, N. Abrams, B. A. Lewis, L. I. Halaoui, T. E. Mallouk, K. D. Benkstein, J. van de Lagemaat and A. J. Frank, J. Am. Chem. Soc., 2003, 125, 6306.

19 T. Toyoda and Q. Shen, J. Phys. Chem. Lett., 2012, 3, 1885.

20 J.-H. Shin, J.-H. Kang, W.-M. Jin, J. H. Park, Y.-S. Cho and J. H. Moon, Langmuir, 2010, 27, 856.

21 J.-H. Shin and J. H. Moon, Langmuir, 2011, 27, 6311.

22 C.-H. Yip, Y.-M. Chiang and C.-C. Wong, J. Phys. Chem. C, 2008, 112, 8735.

23 E. S. Kwak, W. Lee, N. G. Park, J. Kim and H. Lee, Adv. Funct. Mater., 2009, 19, 1093.

24 S. C. Pilcher and W. T. Ford, Macromolecules, 1998, 31, 3454. 25 Y. N. Xia, B. Gates, Y. D. Yin and Y. Lu, Adv. Mater., 2000, 12, 693. 26 J. W. Galusha, C.-K. Tsung, G. D. Stucky and M. H. Bartl, Chem. Mater., 2008, 20, 4925.

27 M. C. Orilall, N. M. Abrams, J. Lee, F. J. DiSalvo and U. Wiesner, J. Am. Chem. Soc., 2008, 130, 8882.

28 A. Stein, F. Li and N. R. Denny, Chem. Mater., 2008, 20, 649. 29 S. Sokolov, B. Paul, E. Ortel, A. Fischer and R. Kraehnert, Langmuir, 2011, 27, 1972. 
30 A. B. D. Nandiyanto, F. Iskandar and K. Okuyama, Chem. Eng. J., 2009, 152, 293.

31 G. Ruani, C. Ancora, F. Corticelli, C. Dionigi and C. Rossi, Sol. Energy Mater. Sol. Cells, 2008, 92, 537.

32 B. Hatton, L. Mishchenko, S. Davis, K. H. Sandhage and J. Aizenberg, Proc. Natl. Acad. Sci. U. S. A., 2010, 107, 10354.

33 C. Dionigi, P. Greco, G. Ruani, M. Cavallini, F. Borgatti and F. Biscarini, Chem. Mater., 2008, 20, 7130.

34 M.-A. Kim, Y. G. Seo, S.-S. Lee, W. Lee and H. Lee, J. Nanosci. Nanotechnol., 2012, 12, 3247.

35 P. C. A. Alberius, K. L. Frindell, R. C. Hayward, E. J. Kramer, G. D. Stucky and B. F. Chmelka, Chem. Mater., 2002, 14, 3284.

36 E. L. Crepaldi, G. J. d. A. A. Soler-Illia, D. Grosso, F. Cagnol, F. Ribot and C. Sanchez, J. Am. Chem. Soc., 2003, 125, 9770.

37 D. Fattakhova-Rohlfing, M. Wark, T. Brezesinski, B. M. Smarsly and J. Rathouský, Adv. Funct. Mater., 2007, 17, 123.

38 M. Rawolle, K. Sarkar, M. A. Niedermeier, M. Schindler, P. Lellig, J. S. Gutmann, J.-F. Moulin, M. Haese-Seiller, A. S. Wochnik, C. Scheu and P. Müller-Buschbaum, ACS Appl. Mater. Interfaces, 2013, 5(3), 719-729.

39 P. D. Yang, D. Y. Zhao, D. I. Margolese, B. F. Chmelka and G. D. Stucky, Nature, 1998, 396, 152.

40 E. Ortel, A. Fischer, L. Chuenchom, J. Polte, F. Emmerling, B. Smarsly and R. Kraehnert, Small, 2012, 8, 298.

41 Y. Liu, J. M. Szeifert, J. M. Feckl, B. Mandlmeier, J. Rathousky, O. Hayden, D. Fattakhova-Rohlfing and T. Bein, ACS Nano, 2010, 4, 5373.

42 J. M. Szeifert, D. Fattakhova-Rohlfing, D. Georgiadou, V. Kalousek, J. Rathousky, D. Kuang, S. Wenger, S. M. Zakeeruddin, M. Gratzel and T. Bein, Chem. Mater., 2009, 21, 1260.

43 J. M. Szeifert, D. Fattakhova-Rohlfing, J. Rathousky and T. Bein, Chem. Mater., 2012, 24, 659.
44 J. M. Szeifert, J. M. Feckl, D. Fattakhova-Rohlfing, Y. Liu, V. Kalousek, J. Rathousky and T. Bein, J. Am. Chem. Soc., 2010, 132, 12605.

45 S. Guldin, S. Huttner, P. Tiwana, M. C. Orilall, B. Ulgut, M. Stefik, P. Docampo, M. Kolle, G. Divitini, C. Ducati, S. A. T. Redfern, H. J. Snaith, U. Wiesner, D. Eder and U. Steiner, Energy Environ. Sci., 2011, 4, 225.

46 Y. X. Zhang, G. H. Li, Y. C. Wu, Y. Y. Luo and L. D. Zhang, J. Phys. Chem. B, 2005, 109, 5478.

47 D. P. Macwan, P. Dave and S. Chaturvedi, J. Mater. Sci., 2011, 46, 3669.

48 H. Li, Z. Bian, J. Zhu, D. Zhang, G. Li, Y. Huo, H. Li and Y. Lu, J. Am. Chem. Soc., 2007, 129, 8406.

49 E. A. Barringer and H. K. Bowen, J. Am. Ceram. Soc., 1982, 65, C199.

50 C.-C. Wang and J. Y. Ying, Chem. Mater., 1999, 11, 3113.

51 S. Shao, M. Dimitrov, N. Guan and R. Kohn, Nanoscale, 2010, 2, 2054.

52 B. Mandlmeier, J. M. Szeifert, D. Fattakhova-Rohlfing, H. Amenitsch and T. Bein, J. Am. Chem. Soc., 2011, 133, 17274.

53 M. Niederberger, M. H. Bartl and G. D. Stucky, Chem. Mater., 2002, 14, 4364.

54 S. Ito, P. Chen, P. Comte, M. K. Nazeeruddin, P. Liska, P. Pechy and M. Gratzel, Prog. Photovoltaics, 2007, 15, 603.

55 J. Schuster, G. He, B. Mandlmeier, T. Yim, K. T. Lee, T. Bein and L. F. Nazar, Angew. Chem., Int. Ed., 2012, 124, 3651.

56 C. J. Barbé, F. Arendse, P. Comte, M. Jirousek, F. Lenzmann, V. Shklover and M. Grätzel, J. Am. Ceram. Soc., 1997, 80, 3157.

57 S. Ito, T. N. Murakami, P. Comte, P. Liska, C. Grätzel, M. K. Nazeeruddin and M. Grätzel, Thin Solid Films, 2008, 516, 4613.

58 H. Němec, V. Zajac, I. Rychetsk, D. Fattakhova-Rohlfing, B. Mandlmeier, T. Bein, Z. Mics and P. Kužel, IEEE Trans. Terahertz Sci. Technol., 2013, 3, 302. 